\title{
Astrometric photographic observations of Helene $e^{\star, \star \star}$
}

\author{
C.H. Veiga and R. Vieira Martins \\ Observatório Nacional, Rua Gal. José Cristino 77, 20921-400 Rio de Janeiro, Brazil \\ e-mail: cave@on.br or rvm@on.br
}

Received November 16, 1999; accepted January 19, 2000

\begin{abstract}
Astrometric photographic positions of the Saturnian satellite Helene obtained from 22 plates taken in 6 nights are presented. The observations are distributed between 5 missions in 1985 and 1987. All positions are compared with those calculated by Jacobson (1999). The residuals have mean and standard deviation smaller than 0.25 and $0 . \prime 30$ respectively.
\end{abstract}

Key words: astrometry — planets and satellites — Saturn

\section{Introduction}

Helene, the faint Lagrangian satellite of Saturn-Dione, was discovered in 1980 during the Earth ring-plane crossing (Lecacheux et al. 1980). Its visual magnitude is about 17 . Due to its faintness and its proximity to scattered light from the planet and rings, this satellite is very difficult to observe. Therefore the number of astrometric positions of Helene is not very large and the positions are not very accurate.

After its discovery, Helene was observed in 1980-81 (Reitsema 1981) and from 1981 to 1985 by Veillet (Oberti et al. 1989). In total, there are 233 published positions of this satellite. Some observations made in 1992 and 1994 are informed by Rodhe \& Pascu (1993, 1994).

An analytical theory for the motion of Helene was presented by Oberti (1990). After 1995 a numerical integration of this longitude: $-3^{\mathrm{h}} 02^{\mathrm{m}} 19^{\mathrm{s}}$, latitude: $-22^{\circ} 32^{\prime} 04^{\prime \prime}$ and altitude: $1872 \mathrm{~m}$. The plates are the same used in our observations of the large satellites of Saturn. All plates were hypersensitized and the exposure times have varied from 8 to 12 minutes. No filters or masks on the plates

Send offprint requests to: C.H. Veiga

* Based on observations made at Laboratório Nacional de Astrofísica/CNPq/MCT-Itajubá-Brazil.

** Table 1 is also available in electronic form at the CDS via anonymous ftp to cdsarc.u-strasbg.fr (130.79.128.5) or via http://cdsweb.u-strabg.fr/Abstract.html
Table 1. Observed positions of Helene. For each plate we present the year, month, day, and decimal fractions of TDT days, corresponding to the mean instant of the observation. The differential topocentric coordinates, $X(\Delta \alpha \cos \delta)$ and $Y(\Delta \delta)$, are referred to Titan. The reference system is defined by the mean equator and equinox J2000

\begin{tabular}{|c|c|c|c|c|c|}
\hline \multicolumn{2}{|c|}{ Date } & \multirow{2}{*}{$\begin{array}{c}\Delta \alpha \cos \delta \\
\prime \prime\end{array}$} & \multirow{2}{*}{$\begin{array}{l}\Delta \delta \\
\prime \prime\end{array}$} & \multirow{2}{*}{$\begin{array}{c}\overline{(\mathrm{O}-\mathrm{C})_{x}} \\
\prime \prime\end{array}$} & \multirow{2}{*}{$\overline{(\mathrm{O}-\mathrm{C})_{2}}$} \\
\hline Y M & $\mathrm{D}$ & & & & \\
\hline 19853 & 0.15000 & +120.204 & +38.768 & +0.556 & -0.142 \\
\hline 19853 & 0.15903 & +121.089 & +39.152 & +0.582 & +0.082 \\
\hline 19853 & 0.16806 & +121.384 & +39.444 & -0.010 & +0.224 \\
\hline 19853 & 0.17431 & +121.861 & +39.410 & -0.148 & +0.100 \\
\hline 19853 & 0.18681 & +123.582 & +39.301 & +0.313 & -0.199 \\
\hline 19853 & 0.19514 & +124.092 & +39.574 & -0.050 & -0.046 \\
\hline 19853 & 0.20417 & +125.415 & +39.236 & +0.314 & -0.514 \\
\hline 19853 & 0.21597 & +125.969 & +39.963 & -0.406 & +0.063 \\
\hline 19853 & 0.22847 & +127.860 & +40.205 & +0.097 & +0.155 \\
\hline 19854 & 16562 & +144.153 & -75.611 & +0.194 & +0.349 \\
\hline 19854 & 9.17014 & +026.304 & +72.127 & +0.015 & -0.443 \\
\hline 19854 & 9.17674 & +027.193 & +72.273 & +0.200 & -0.527 \\
\hline 19854 & 9.19375 & +029.734 & +73.390 & +0.891 & +0.010 \\
\hline 19854 & 9.20347 & +029.570 & +73.868 & -0.377 & +0.148 \\
\hline 19856 & 1.96319 & -218.385 & +40.767 & +0.731 & -0.273 \\
\hline 19856 & 1.97222 & -218.073 & +41.629 & +0.380 & -0.071 \\
\hline 19856 & 1.98194 & -216.844 & +42.227 & +0.890 & -0.183 \\
\hline 19856 & 1.99097 & -216.915 & +42.803 & +0.113 & -0.257 \\
\hline 19877 & 6.92083 & -119.697 & -00.473 & +0.264 & -0.083 \\
\hline 19877 & 6.92986 & -119.854 & -00.917 & +0.065 & -0.297 \\
\hline 19877 & 6.94792 & -120.622 & -01.161 & -0.730 & -0.101 \\
\hline 19878 & 4.93889 & -092.083 & -57.410 & +0.034 & -0.220 \\
\hline
\end{tabular}

were used. For some details about the observations see Paper I.

The images of the satellite, the stars and the other satellites of Saturn were digitized with the PDS 1010A of the Observatório Nacional-Brazil. For the images scans a square slit with $20 \mathrm{~m} \mu$ of side was used. To find the center of these images, the ASTROL routines package (Colas \& Serrau 1993) was employed and to determine each center, a two-dimensional Gaussian fitting on a small circular area around the image in which the background was removed 


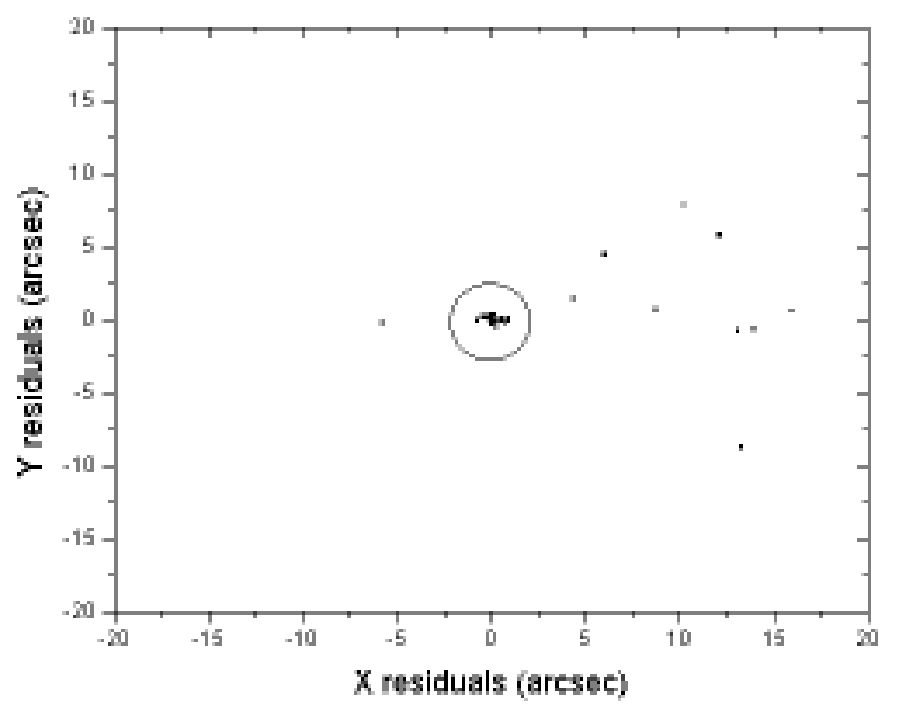

Fig. 1. The observed minus calculated positions of our measured positions on the $x$ and $y$ directions. It can be observed a cluster of 22 positions near the origin. The other 10 positions appear scattered over the plane. Only the central cluster positions were considered in this paper

by a second-degree polynomial, was used. The errors upon the centering procedure were smaller than 0 "'03 for Helene.

For the astrometric calibration we used the method presented in Paper I. The observed positions of Helene are referred to Titan in the reference system J2000. In Paper I, it can be seen that the positions of the large satellites of Saturn have errors smaller than 0 ". 2 when their positions are compared with TASS1.7 (Vienne \& Duriez 1998). Therefore, it is considered that the errors induced by the reduction procedure are smaller than 0 "' 2 . In Table 1, our positions of Helene related to Titan are presented.

The theoretical positions for Helene and Titan were provided by Jacobson from JPL (Jacobson 1999). We inspected all our plates of the Saturnian system (Paper I) in order to identify images of any faint image in the regions around the Dione's Lagrangian points. We found only 32 measurable objects in these plates.

To select the images of Helene from the 32 candidates measured, we compared these observed positions with the theoretical one. We can observe in a plot (Fig. 1) where the axes are the $(\mathrm{O}-\mathrm{C})_{x}$ and $(\mathrm{O}-\mathrm{C})_{y}$ that there is a cluster of points in the neighbohood of the origin, while the other points are spread over the plot. We considered only the positions belonging to the central cluster. These positions of the candidates to Helene images were also compared with the positions of stars (with $B$ and $R$ magnitudes smaller than 20) from the USNO A2.0 Catalog (Monet et al. 1998) in the same field, in order to insure that they are not stellar.

The residuals for our observed positions of Helene compared with theoretical positions have $\bar{x}=0$ ". 270 $\bar{y}=-0^{\prime \prime} 223, \sigma_{x}=0^{\prime \prime} .320$ and $\sigma_{y}=0^{\prime \prime} .278$. In Fig. 2 the
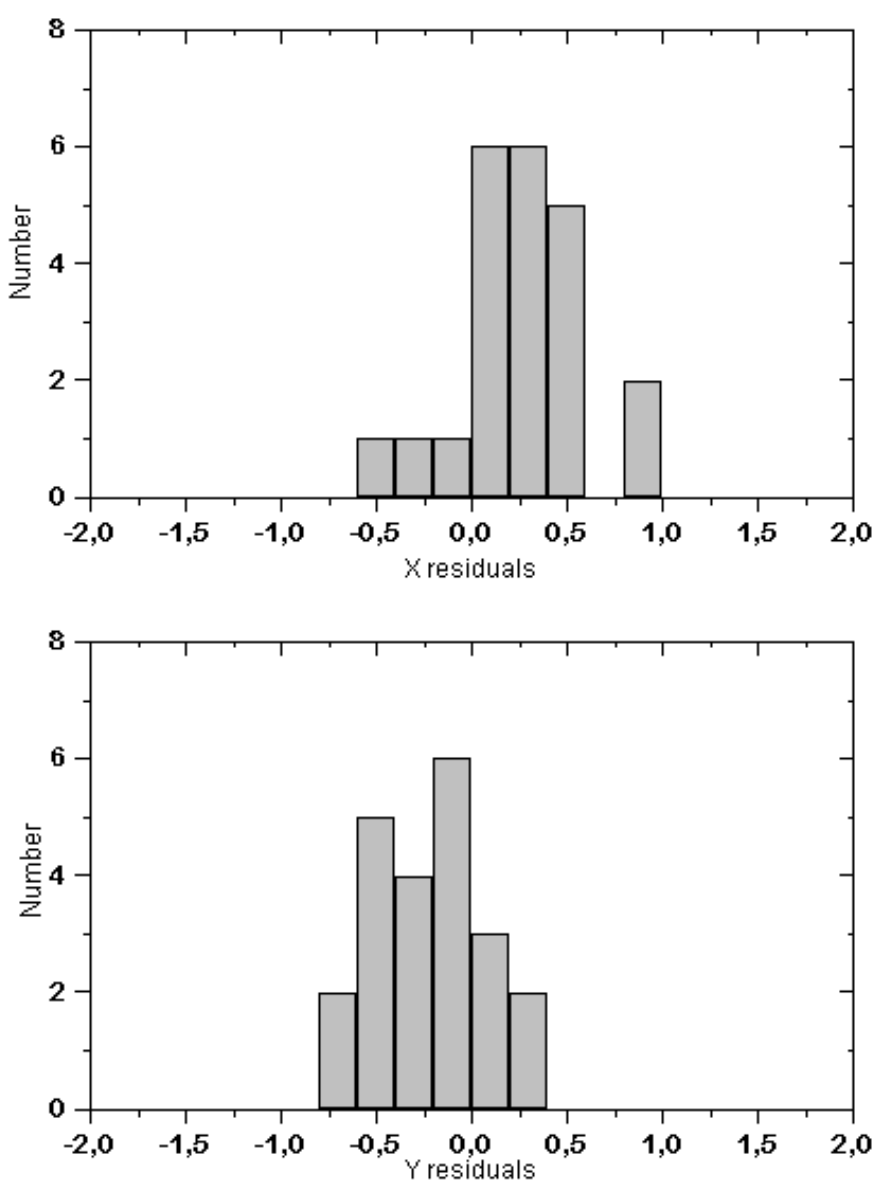

Fig. 2. Histogram for the residuals of Helene referred to Titan $(X=\Delta \alpha \cos \delta$ and $Y=\Delta \delta)$

histogram for the residuals $x$ and $y$, is presented. It can be noted that the standard deviation in the $x$ direction is larger than for the $y$ direction. This is due to guiding problems.

\section{Comments}

We presented 22 astrometric photographic positions of Helene. The observations of this satellite is very difficult. However, using special procedures and CCD detectors, we observed in the last years this satellite and also the Tethys Lagrangian satellites Telesto and Calypso. The results of these observations will be presented in a forthcoming paper.

Acknowledgements. The authors wish to thank Dr. R.A. Jacobson for the theoretical positions of Helene provided and the CNPq-Brazil for partial support of this work. C.H. Veiga would like to thank State of Rio de Janeiro Science Foundation - FAPERJ for providing computational facilities for this project. 


\section{References}

Colas F., Serrau M., 1993, Astrol and Interpol version 3.10, Édition du Bureau des Longitudes

Jacobson J.A., 1995, BAAS, 27-3, 1202

Jacobson J.A., 1999 (personal communication)

Lecacheux J., Lasques P., Vapillon L., Auge A., Despiau R., 1980, Icarus 43, 111

Monet, et al., 1998, USNO A2.0 A catalog of astrometric standards, U.S. Naval Observatory

Oberti P., 1990, A\&A 228, 275

Oberti P., Veillet C., Catullo V., 1989, A\&AS 80, 289

Reitsema H.J., 1981, Icarus 48, 23

Rohde J.R., Pascu D., 1993, BAAS 25-3, 1235

Rohde J.R., Pascu D., 1994, BAAS 26-2, 862

Veiga C.H., Vieira Martins R., 1999, A\&AS 139, 305

Vienne A., Duriez L., 1995, A\&A 297, 588 